\title{
O estágio de manutenção para o exercício está associado à uma melhor percepção de diferentes indicadores de saúde na adolescência
}

\author{
Maintenance to exercise is associated to adolescents' perceived health
}

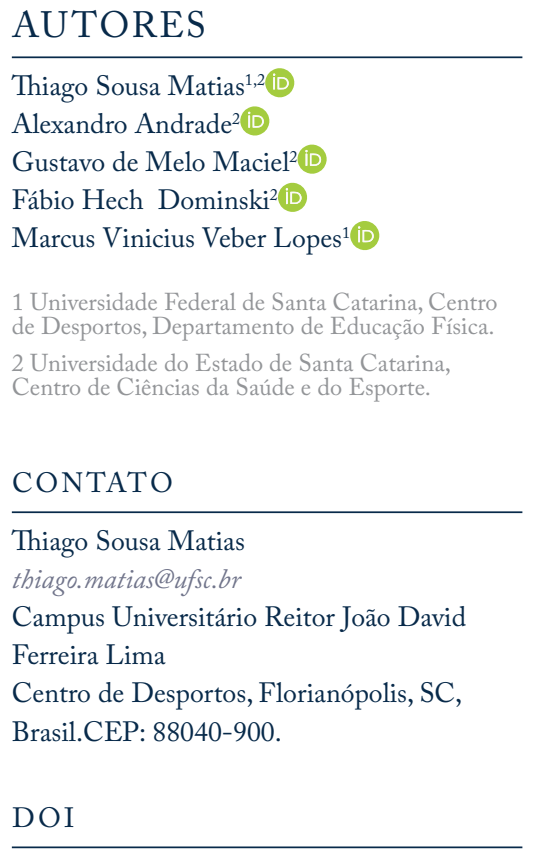

10.12820/rbafs.v.22n5p479-85

\section{(1) $\Theta \Theta$}

\begin{abstract}
RESUMO
O objetivo deste estudo foi investigar a associação entre diferentes estágios de mudança do comportamento para o exercício e a percepção de indicadores de saúde em estudantes adolescentes. Participaram 662 adolescentes de 14 a 19 anos, distribuídos proporcionalmente nos 12 distritos de Florianópolis/SC. Foram utilizados: questionários de caracterização socioeconômica, condições de saúde e avaliação dos estágios de mudança de comportamento para o exercício. Observou-se que o estágio de manutenção para o exercício esteve associado às percepções de saúde. Rapazes em manutenção, apresentaram maiores chances de avaliarem positivamente sua saúde (OR=3,65; IC95\%: 1,62-8,25). Entre as moças, o estágio de manutenção para a prática de exercícios foi associado à satisfação com o peso corporal (OR=1,68; IC95\%: 1,01-2,80) e ao melhor autocontrole do estresse $(\mathrm{OR}=1,88$; IC95\%: 1,03-3,42). Rapazes e moças no estágio de manutenção para a prática de exercícios apresentaram chances reduzidas de perceberem-se frequentemente doentes (rapazes: $O R=0,32$; IC95\%: 0,16-0,63; moças: OR=0,57; IC95\%: 0,33-0,97). Adolescentes em manutenção para o exercício físico apresentaram melhores percepções de saúde comparados aqueles que não praticaram atividades físicas. A percepção positiva esteve associada ao tempo de exposição ao exercício, pois não foram observadas associações em adolescentes no estágio de ação.
\end{abstract}

Palavras-chave: Atividade motora; Exercício; Estudantes; Percepção de peso; Sono; Estresse psicológico. ABSTRACT

This study aimed to analyze the association between the stages of exercise behaviorand perceived health of adolescent students. The sample was composed by 662 adolescents aged between 14 to 19 years old, proportionally distributed among 12 districts of Florianopolis/SC/Brazil. Health conditions (1) and (2) Stages of exercise behavior change questionnaires were used. We observed that maintenance stage of physical exercise was associated with perceived health. Boys in maintenance were more likely to positively assess their health (OR: 3.65; CI95\%: 1.62-8.25). Among girls, the maintenance for exercise was associated with body weight satisfaction(OR: 1.68; CI95\%: 1.01-2.80) and better stress management (OR: 1.88; CI95\%: 1.03-3.42). Boys and girls in the maintenance stage for exercise are less likely to perceive themselves sick (boys: OR: 0.32; CI95\%: 0.16-0.63; girls: OR:0.57; IC95\%:0.33-0.97). We conclude that adolescents in maintenance for exercise showed a better perception of their health conditions compared to those who did not engage in physical activities regularly. Adaptive benefits of exercise seem to appear as adolescent maintain physical activities over time.

Keywords: Motor activity; Exercise; Students; Weight perception; Sleep; Stress, psychological.

\section{Introdução}

A prática de atividade física tem sido associada à inúmeros benefícios de saúde, com implicações importantes para a manutenção da saúde física, mental e social ${ }^{1}$. Esses benefícios quando percebidos por crianças e adolescentes apresentam maiores chances de perdurarem para a vida adulta, impactando positivamente no bem-estar individual no curso da vida ${ }^{2}$. Neste contexto, a prática regular de exercícios tem sido observada como importante na formação de conceitos relativos à saúde percebida ${ }^{3}$.
Alguns dos benefícios da prática regular de atividade física estão relacionados à melhora na qualidade de sono $^{4}$, no controle do estresse $\mathrm{e}^{5}$ na percepção de indicadores de imagem corporal ${ }^{6}$. Entretanto, apesar dos referidos benefícios, não é claro se adolescentes conseguem perceber estes resultados sobre a saúde considerando que as estratégias para a promoção da atividade física nesta população ainda são frágeis ${ }^{1}$ e a prevalência de adolescentes insuficientemente ativos no Brasil $(60,8 \%)$ é alta nos diferentes domínios de atividade física ${ }^{7}$. 
A prática de exercícios tem sido majoritariamente investigada por meiode medidas objetivas, explorando principalmente o volume e a intensidade das atividades ${ }^{8}$, o que pode implicar em alguns vieses na relação com indicadores de saúde. Neste trabalho, assume-se que os aspectos benéficos do exercício para a saúde vão além das consequências relacionadas ao gasto energético e que fatores psicológicos, sociais e cognitivos podem apresentar informações adicionais nas explicações relacionadas à saúde 9 .

Assume-se que a participação em exercícios é também um processo que representa comportamentos motivacionais e sugere diferentes percepções. Neste caso, implica numa ação intencional que se revela ao longo do tempo através de uma série de estágios de mudança do comportamento $(\mathrm{EMC})^{10}$.O Modelo Transteorético classifica os indivíduos em cinco diferentes estágios de mudança de comportamento (pré-contemplação, contemplação, preparação, ação e manutenção) permitindo identificar não só o comportamento atual, mas também a intenção de mudar o hábito ${ }^{11}$. Ainda, o EMC permite entender que a relação entre o exercício e o estabelecimento de conceitos é consequente do seu desenvolvimento ao longo do tempo ${ }^{12}$. Num estudo recente com universitários foi observado que aqueles em manutenção para o exercício são significativamente mais autodeterminados comparados aos estágios de preparação e ação, indicando que neste estágio as experiências são mais significativas e duradouras ${ }^{13}$.

Sugere-se que as diferentes características motivacionais do exercício podem determinar a adoção e/ ou manutenção do comportamento, relacionando-se diferentemente com o comportamento e a percepção de saúde ${ }^{11}$. Estar num estágio específico de comportamento para o exercício pode dar suporte ou não para perceber o estado de saúde ${ }^{2}$. Estes pressupostos foram investigados em pessoas com depressão e foi observado que a manutenção para o exercício é conduzida com alto grau de autonomia, favorecendo alterações perceptivas importantes na relação com a saúde ${ }^{14}$.

Diante disso, observa-se que os pressupostos do modelo transteórico, como a diferenciação de pessoas em ação e manutenção para a prática, tem sido positivamente associado com a aquisição de comportamentos relacionados à saúde ${ }^{13,14}$. Entretanto, poucas são as evidências relativas ao conjunto de alterações no âmbito das percepções. Sendo assim, objetivo deste estudo foi investigar a associação entre diferentes estágios de mudança do comportamento para o exercício e a percepção de indicadores de saúde de estudantes adolescentes.

\section{Métodos}

Este estudo caracteriza-se como de delineamento transversal e de base populacional. Trata-se de análise secundária do projeto "Aspectos psicossociais e motivacionais para a prática de atividade física na adolescência” realizado em 2010. Foram elegíveis para o estudo estudantes do ensino médio matriculados na instituição pública de ensino de maior porte em Santa Catarina. A amostra foi constituída por adolescentes de 14 a 19 anos $(15 \pm 1,0)$, de ambos os sexos, estudantes do período matutino e vespertino.

Utilizou-se dos dados do último Censo do IBGE de 2010/2011 para o cálculo amostral. Considerou-se um erro máximo de cinco pontos percentuais e confiabilidade de 99,0\%, resultando em tamanho amostral final de 657 adolescentes distribuídos proporcionalmente entre os 12 distritos do município de Florianópolis (Santa Catarina). Ao final dos procedimentos de coleta de dados, 662 adolescentes participaram do estudo.

Os indicadores de saúde - autoavaliação da saúde, frequência que apresenta doenças, satisfação com o peso corporal, qualidade do repouso e sono e autocontrole do estresse - foram mensurados por meio do instrumento "Questionário de autoavaliação do estilo de vida" adaptado ${ }^{15}$. Todos os indicadores de percepção de saúde são provenientes de questões cujas categorias de resposta se apresentam em escala do tipo Likert, com cinco itens. Para fins de análise, a autoavaliação de saúde foi avaliada como "negativa" ("péssima", "ruim" e "regular") e "positiva" ("boa" e "excelente"); a frequência que apresenta doenças foi categorizada como "raramente" ("não fico doente" e "poucas vezes") e "frequentemente" ("às vezes", "muitas vezes" e "quase sempre”); a satisfação com o peso foi tratada como "insatisfeito" ("nada satisfeito" e "pouco satisfeito") e "satisfeito" ("satisfeito", "muito satisfeito" e "extremamente satisfeito"); a qualidade do sono e autocontrole do estresse foram avaliados em "ruim" ("péssimo", "ruim” e "regular") e "bom" ("bom"e "excelente").

Os dados referentes ao EMC para a prática de exercícios foram obtidos por meio de questionário ${ }^{16} \mathrm{e}-$ laborado a partir do modelo transteorético de mudança de comportamento ${ }^{11}$. O instrumento possibilita a classificação dos indivíduos conforme sua pretensão, execução e permanência na prática de exercícios físicos regulares. As categorias de resposta referentes aos estágios de pré-contemplação, contemplação e preparação foram agrupadas em "não pratica exercícios físicos". Já as categorias de resposta referentes aos estágios de ação 
e manutenção foram mantidas conforme mensuradas. A medida da classe econômica foi obtida por meio da classificação proposta pela Associação Brasileira de Empresas em Pesquisa (ABEP). A classificação utiliza de informações quanto aos itens existentes na residência do participante e da escolaridade do chefe da família. As classes identificadas foram agrupadas em classe econômica "alta" (classe A1 e A2), "média" (classe B1 e B2) e "baixa" (classe C1, C2, D e E), em virtude da distribuição dos dados.

As análises estatísticas foram conduzidas no pacote estatístico Stata 13. Utilizou-se de estatística descritiva (frequência absoluta e relativa) para caracterização da amostra de acordo com as variáveis investigadas. As associações entre o EMC e os indicadores de saúde foram avaliadas por meio de modelos de regressão logística binária bruta e ajustada, dos quais obtiveram-se os valores de odds ratio (OR) e intervalos de confiança de $95 \%$ (IC95\%). As categorias relativas aos indicadores negativos de saúde, bem como a categoria "não pratica exercícios físicos" do EMC foram tratadas como categorias de referência nos modelos de regressão. Adotou-se de entrada simultânea das variáveis no modelo ajustado, com ajuste para as variáveis sociodemográficas turno de estudo, idade, trabalho e nível socioeconômico.

Os estudantes participaram do estudo mediante entrega do Termo de Consentimento Livre e Esclarecido, devidamente assinado pelos pais ou responsáveis, quando menores de 18 anos de idade. A pesquisa foi autorizada pelo Comitê de Ética em Pesquisa em Seres Humanos (CEP) da Universidade do Estado de Santa Catarina ( $n^{\circ}$ de referência 134/09).

\section{Resultados}

Do total de 662 adolescentes que participaram do estudo, 659 responderam às questões referentes aos indicadores de saúde. A maior parte dos adolescentes era do sexo feminino $(55,7 \%)$, estudava no período vespertino $(52,6 \%)$, não trabalhava (80,4\%), e pertencia à classe econômica média (72,2\%). As características sociodemográficas, conforme os sexos, estão disponíveis na Tabela 1.

Ao analisar os indicadores de percepção de saúde e o EMC para prática de exercícios físicos em adolescentes (Tabela 2), observou-se que os rapazes avaliavam positivamente sua saúde $(84,1 \%)$, raramente percebiam-se doentes $(74,5 \%)$, eram satisfeitos com seu peso corporal $(69,7 \%)$ e estavam no estágio de manutenção da prática de exercícios físicos (62,9\%). As moças, por sua vez, avaliavam positivamente sua saúde $(74,4 \%)$, raramente percebiam-se doentes $(62,2 \%)$, tinham autocontrole de estresse prejudicado $(73,7 \%)$ e mantinham a prática de exercícios $(14,4 \%$ no estágio de ação; $37,9 \%$ no estágio de manutenção).

Quando analisada a associação do EMC para a prática de exercícios físicos com os indicadores de percepção de saúde (Tabela 3), observou-se na análise ajustada, que rapazes praticantes de exercícios físicos a mais de seis meses (estágio de manutenção) apresentaram maiores chances de avaliarem positivamente sua saúde $(\mathrm{OR}=3,65$; IC95\%: 1,62-8,25). Já entre as moças, o estágio de manutenção para a prática de exercícios foi associado a satisfação com o peso corporal $(\mathrm{OR}=1,68$; IC95\%: 1,01-2,80) e ao melhor autocontrole do estresse (OR=1,88; IC95\%: 1,03-3,42). Rapazes e moças no estágio de manutenção para a prática de exercícios apresentaram chances reduzidas de perceberem-se frequentemente doentes (rapazes: OR=0,32; IC95\%:

Tabela 1 - Características sociodemográficas da amostra de acordo com os sexos. Florianópolis, Santa Catarina, 2010 (n=653).

\begin{tabular}{|c|c|c|c|c|}
\hline \multirow{3}{*}{$\begin{array}{l}\text { Variáveis } \\
\text { Idade (média } \pm \text { dp) }\end{array}$} & \multicolumn{2}{|c|}{ Rapazes $(n=290)$} & \multicolumn{2}{|c|}{ Moças $(n=363)$} \\
\hline & \multicolumn{2}{|c|}{$16,0 \pm 1,1$} & \multicolumn{2}{|c|}{$15,8 \pm 1,0$} \\
\hline & $\%$ & IC $95 \%$ & $\%$ & IC95\% \\
\hline \multicolumn{5}{|l|}{ Turno de estudo } \\
\hline Matutino & 47,5 & $(41,8 ; 53,4)$ & 47,1 & $(41,9 ; 52,3)$ \\
\hline Vespertino & 52,5 & $(46,6 ; 58,2)$ & 52,9 & $(47,7 ; 58,1)$ \\
\hline \multicolumn{5}{|l|}{ Atividade profissional } \\
\hline Trabalha & 24,3 & $(19,6 ; 29,6)$ & 16,1 & $(12,6 ; 20,2)$ \\
\hline Não trabalha & 75,7 & $(70,4 ; 80,4)$ & 83,9 & $(79,8 ; 87,4)$ \\
\hline \multicolumn{5}{|l|}{ Classe econômica } \\
\hline Alta & 10,5 & $(7,4 ; 14,7)$ & 9,0 & $(6,4 ; 12,5)$ \\
\hline Média & 76,8 & $(71,4 ; 81,4)$ & 68,7 & $(63,6 ; 73,4)$ \\
\hline Baixa & 12,7 & $(9,2 ; 17,2)$ & 22,3 & $(18,2 ; 27,0)$ \\
\hline
\end{tabular}

Nota: dp: desvio padrão; IC95\%: intervalos de confiança de 95\%. 
Tabela 2 - Indicadores de percepção de saúde e estágio de mudança de comportamento (EMC) para exercícios físicos em rapazes e moças, Florianópolis, Santa Catarina, $2010(n=653)$.

\begin{tabular}{|c|c|c|c|c|}
\hline \multirow{2}{*}{ Variáveis } & \multicolumn{2}{|c|}{ Rapazes $(n=290)$} & \multicolumn{2}{|c|}{ Moças $(n=363)$} \\
\hline & $\%$ & IC95\% & $\%$ & IC95\% \\
\hline \multicolumn{5}{|l|}{ Autoavaliação de saúde } \\
\hline Negativa & 15,86 & $(12,08 ; 20,55)$ & 25,62 & $(21,38 ; 30,38)$ \\
\hline Positiva & 84,14 & $(79,45 ; 87,92)$ & 74,38 & $(69,62 ; 78,62)$ \\
\hline \multicolumn{5}{|c|}{ Frequência que apresenta doenças } \\
\hline Raramente & 74,48 & $(69,13 ; 79,19)$ & 62,15 & $(57,03 ; 67,02)$ \\
\hline Frequentemente & 25,52 & $(20,81 ; 30,87)$ & 37,85 & $(32,98 ; 42,97)$ \\
\hline \multicolumn{5}{|c|}{ Satisfação com o peso corporal } \\
\hline Insatisfeito & 30,34 & $(25,31 ; 35,90)$ & 48,90 & $(43,76 ; 54,05)$ \\
\hline Satisfeito & 69,66 & $(64,10 ; 74,69)$ & 51,10 & $(45,95 ; 56,24)$ \\
\hline \multicolumn{5}{|c|}{ Qualidade do sono e repouso } \\
\hline Ruim & 45,52 & $(39,85 ; 51,30)$ & 46,01 & $(40,92 ; 51,17)$ \\
\hline Boa & 54,48 & $(48,70 ; 60,15)$ & 53,99 & $(48,83 ; 59,08)$ \\
\hline \multicolumn{5}{|l|}{ Autocontrole de estresse } \\
\hline Ruim & 49,66 & $(43,91 ; 55,41)$ & 73,68 & $(68,88 ; 77,98)$ \\
\hline Bom & 50,34 & $(44,59 ; 56,09)$ & 26,32 & $(22,02 ; 31,12)$ \\
\hline \multicolumn{5}{|l|}{ EMC } \\
\hline Não pratica exercícios & 23,90 & $(19,18 ; 29,35)$ & 47,71 & $(42,32 ; 53,15)$ \\
\hline Ação & 13,24 & $(09,69 ; 17,82)$ & 14,37 & $(10,96 ; 18,62)$ \\
\hline Manutenção & 62,87 & $(56,95 ; 68,43)$ & 37,92 & $(32,80 ; 43,32)$ \\
\hline
\end{tabular}

Nota: IC95\% =intervalos de confiança de 95\%. ECM: Estágio de Mudança de Comportamento.

Tabela 3 - Odds Ratio (OR) bruta e ajustada e intervalos de confiança de 95\% (IC95\%) para o estágio de mudança de comportamento (EMC) para a prática de exercícios físicos associado a indicadores de percepção de saúde em rapazes e moças, Florianópolis, Santa Catarina, 2010 ( $\mathrm{n}=653$ ).

\begin{tabular}{|c|c|c|c|c|c|c|c|c|c|c|c|c|}
\hline \multirow{3}{*}{ Variáveis } & \multicolumn{6}{|c|}{ Rapazes $(n=290)$} & \multicolumn{6}{|c|}{$\operatorname{Moças~}(n=363)$} \\
\hline & \multicolumn{3}{|c|}{ Bruta } & \multicolumn{3}{|c|}{ Ajustada } & \multicolumn{3}{|c|}{ Bruta } & \multicolumn{3}{|c|}{ Ajustada } \\
\hline & OR & IC95\% & $\mathrm{p}$ & OR & IC95\% & $\mathrm{p}$ & OR & IC95\% & $\mathrm{p}$ & OR & IC95\% & $\mathrm{p}$ \\
\hline \multicolumn{13}{|l|}{ Desfecho 1: Autoavaliação positiva de saúde } \\
\hline Não pratica & 1,0 & & & 1,0 & & & 1,0 & & & 1,0 & & \\
\hline Ação (pratica a menos de 6 meses) & 1,15 & $(0,45 ; 2,91)$ & 0,77 & 1,09 & $(0,41 ; 2,93)$ & 0,86 & 1,16 & $(0,56 ; 2,40)$ & 0,68 & 1,28 & $(0,60 ; 2.73)$ & 0,53 \\
\hline Manutenção (pratica a mais de 6 meses) & 3,71 & $(1,76 ; 7,84)$ & $0,00^{*}$ & 3,65 & $(1,62 ; 8,25)$ & $0,00^{*}$ & 1,83 & $(1,05 ; 3,21)$ & $0,03^{*}$ & 1,77 & $(0,98 ; 3.20)$ & 0,06 \\
\hline \multicolumn{13}{|l|}{ Desfecho 2: Frequentemente apresenta doenças } \\
\hline Não pratica & 1,0 & & & & & & 1,0 & & & & & \\
\hline Ação (pratica a menos de 6 meses) & 0,70 & $(0,30 ; 1,65)$ & 0,42 & 0,73 & $(0,30 ; 1,80)$ & 0,50 & 0,44 & $(0,07 ; 2,74)$ & 0,38 & 1,09 & $(0,55 ; 2,14)$ & 0,80 \\
\hline Manutenção (pratica a mais de 6 meses) & 0,32 & $(0,17 ; 0,61)$ & $0,00^{*}$ & 0,32 & $(0,16 ; 0,63)$ & $0,00^{*}$ & 0,59 & $(0,13 ; 2,67)$ & 0,49 & 0,57 & $(0,33 ; 0,97)$ & $0,03^{*}$ \\
\hline \multicolumn{13}{|l|}{ Desfecho 3: Satisfação com o peso corporal } \\
\hline Não pratica & 1,0 & & & 1,0 & & & 1,0 & & & & & \\
\hline Ação (pratica a menos de 6 meses) & 0,77 & $(0,33 ; 1,77)$ & 0,53 & 0,52 & $(0,21 ; 1,27)$ & 0,15 & 0,99 & $(0,51 ; 1,90)$ & 0,97 & 1,04 & $(0,53 ; 2,04)$ & 0,91 \\
\hline Manutenção (pratica a mais de 6 meses) & 1,63 & $(0,88 ; 3,01)$ & 0,12 & 1,35 & $(0,70 ; 2,63)$ & 0,37 & 1,64 & $(1,02 ; 2,65)$ & $0,04^{*}$ & 1,68 & $(1,01 ; 2,80)$ & $0,05^{*}$ \\
\hline \multicolumn{13}{|l|}{ Desfecho 4: Boa qualidade do sono e repouso } \\
\hline Não pratica & 1,0 & & & 1,0 & & & 1,0 & & & & & \\
\hline Ação (pratica a menos de 6 meses) & 1,55 & $(0,68 ; 3,52)$ & 0,29 & 1,41 & $(0,59 ; 3,36)$ & 0,44 & 0,86 & $(0,44 ; 1,65)$ & 0,64 & 0,73 & $(0,37 ; 1,44)$ & 0,36 \\
\hline Manutenção (pratica a mais de 6 meses) & 1,67 & $(0,94 ; 2,96)$ & 0,08 & 1,66 & $(0,90 ; 3,07)$ & 0,10 & 0,99 & $(0,62 ; 1,60)$ & 0,98 & 0,83 & $(0,49 ; 1,38)$ & 0,46 \\
\hline \multicolumn{13}{|l|}{ Desfecho 5: Bom autocontrole de estresse } \\
\hline Não pratica & 1,0 & & & 1,0 & & & 1,0 & & & & & \\
\hline Ação (pratica a menos de 6 meses) & 1,48 & $(0,65 ; 3,35)$ & 0,35 & 1,45 & $(0,62 ; 3,41)$ & 0,40 & 1,83 & $(0,87 ; 3,85)$ & 0,11 & 2,03 & $(0,94 ; 4,40)$ & 0,07 \\
\hline Manutenção (pratica a mais de 6 meses) & 1,58 & $(0,89 ; 2,80)$ & 0,12 & 1,68 & $(0,92 ; 3,07)$ & 0,09 & 2,08 & $(1,20 ; 3,61)$ & $0,01^{*}$ & 1,88 & $(1,03 ; 3,42)$ & $0,04^{*}$ \\
\hline
\end{tabular}

Nota: *Diferença significativa com nível de significância de 5\%. Ajuste para idade, turno de estudo, nível socioeconômico e trabalho. 
0,16-0,63; moças: OR=0,57; IC95\%: 0,33-0,97). Não foram encontradas associações entre o estágio de ação com os diferentes indicadores de saúde.

\section{Discussão}

O presente estudo teve como objetivo investigar a associação entre diferentes estágios de mudança do comportamento para o exercício e a percepção de indicadores de saúde de estudantes adolescentes. Evidenciou-se que a manutenção da prática de exercícios foi associada a melhor autoavaliação de saúde, menor frequência com que apresenta doenças, satisfação com o peso corporal, e melhor autocontrole de estresse.

No que se refere à autoavaliação de saúde, observou-se que adolescentes envolvidos com a prática regular de atividades físicas apresentaram percepção de saúde positiva comparados aqueles que não praticam. Há evidências na literatura apontando que a prática regular de atividade física na adolescência está associada a melhor percepção, de forma que o perfil de saúde de adolescentes ativos é melhor comparado aos inativos fisicamente ${ }^{3,17}$.

Em estudo conduzido na Espanha, com 1109 adolescentes de 10 a 16 anos, foi observado que adolescentes que não participaram de atividades físicas organizadas tinham dificuldades em avaliar positivamente sua saúde, apenas 36,2\% deles apresentaram uma boa percepção de saúde ${ }^{18}$. Segundo resultados apresentados por Piko ${ }^{19}$, em estudo com estudantes húngaros, os adolescentes que não participaram, ou participaram ocasionalmente, de atividades esportivas tiveram maiores chances (OR=1,66; IC95\%: 1,10-2,99) de perceberem sua saúde prejudicada quando comparados aqueles que participaram de atividades esportivas regularmente. Estes achados foram também evidenciados por Herman et al. ${ }^{17}$, em sua pesquisa com aproximadamente 1,8 milhões de adolescentes canadenses; rapazes e moças fisicamente inativos tiveram maiores chances de perceberem sua saúde como regular ou prejudicada $\left(\mathrm{OR}_{\text {rapazes }}=2,09 ; \mathrm{OR}_{\text {moças }}=1,99\right)$ quando comparados aos fisicamente ativos.

A literatura sugere que as pessoas que estão regularmente engajadas em atividades físicas possuem uma melhor disposição psicológica, isso em função das razões motivacionais intrínsecas que levam as pessoas a manterem o comportamento ${ }^{20}$. Isso significa que, quando no estágio de manutenção para a atividade física, os exercícios são conduzidos por alto grau de apreciação e escolha. Ainda, estar envolvido em atividades físicas ao longo do tempo indica satisfação e prazer na prática ${ }^{21}$. Essas evidências permitem entender a manutenção da atividade física em função das suas consequências motivacionais e afetivas, justificando os resultados positivos relacionados à percepção de saúde. Ao que parece, esse princípio hedônico ligado à manutenção da prática de exercício é um mediador importante nas relações positivas com a saúde, sobretudo a psicossocial ${ }^{5,21}$.

Foi observado que rapazes e moças regularmente engajados na prática de exercícios se percebem menos suscetíveis a doenças. Considera-se que os adolescentes constroem a ideia subjetiva sobre sua saúde conforme múltiplo fatores, entre eles a sua condição específica de saúde; isso significa que a percepção também é mediada pela condição física mais imediata desse indivíduo ${ }^{17}$. A literatura tem apontado benefícios do exercício ao sistema imunológico, que repercute na redução da incidência de infecções bacterianas e virais ${ }^{22}$, além de apresentar-se como fator de proteção para múltiplas doenças ${ }^{23}$.

Observou-se que moças em manutenção para a prática de exercícios físicos percebem-se mais satisfeitas com o peso corporal comparadas aquelas que não praticam exercício eesta relação tem sido confirmada na literatura. A associação do exercício com indicadores de percepção corporal implica numa melhor imagem corporal e por consequência melhor bem-estar psicológico ${ }^{24}$. A meta-analise conduzida por Hausenblas e Fallon ${ }^{25}$ apresenta pontos importantes na associação entre exercício e imagem corporal. Segundo os autores, praticantes de exercício possuem uma percepção mais positiva de imagem corporal comparados aqueles que não fazem exercício ${ }^{25}$. Ainda, em intervenções conduzidas com exercícios, os participantes reportaram melhora na percepção da imagem corporal independente de alterações na composição corporal e aptidão física ${ }^{26}$. Outro conjunto de evidências por meta-análise indica que a atividade física na adolescência está associada ao autoconceito geral sobre a condição física, além de uma melhor percepção da aparência ${ }^{6}$.

É importante considerar que, assim como para a percepção geral de saúde, a associação positiva com o peso corporal só se estabeleceu para os adolescentes em manutenção para o exercício. São poucos os estudos que investigarmos efeitos longitudinais da prática de exercício em indicadores de imagem corporal, entretanto, Matias e colaboradores ${ }^{24}$ observaram que em adolescentes, possuir um histórico relacionado à atividade física está associado com uma melhor percepção com o peso, o que indica que houve um período de manutenção relacionada a prática de exercícios resultando na percepção positiva.

Quando observadas as associações com o estresse, os 
resultados também evidenciaram um melhor controle de estresse entre as moças que praticam exercício a mais de seis meses comparadas às inativas fisicamente. Esses achados dão suporte para relação entre exercício e resposta emocional ${ }^{5}$ e sinaliza que estar regularmente engajado na atividade física pode resultar na mudança de crenças do adolescente para realizar com sucesso algumas atividades, resultando em maior autoeficácia diante dos comportamentos do dia a dia ${ }^{27}$. Suporte social, satisfação e o alcance no cumprimento de metas tem sido associado com melhor bem-estar psicológico ${ }^{28}$ e são características importantes e consequentes da manutenção da prática de exercícios. Observa-se que a atividade física eleva a autoestima e atua como distração para os estressores do dia a dia e impede pensamentos negativos ruminantes, consequentemente, propicia ao indivíduo a condição de ter atitudes e pensamentos mais positivos $^{29}$. Estes benefícios ligados ao melhor controle de estresse parecem estar ligados às sucessivas e positivas respostas emocionais da prática de exercícios ${ }^{5}$.

São sinalizadas algumas limitações metodológicas importantes e as inferências precisam ser interpretadascom cautela. Esses resultados são derivados de um delineamento transversal e a exposição à prática de exercícios foi observada a partir de medidas autorrelatadas. É importante observar que nossas hipóteses são derivadas de um referencial teórico em que os estágios de mudança para o exercício representam disposições motivacionais. Neste caso, as explicações das associações positivas entre exercício e saúde são mediadas por fatores psicossociais, entretanto, variáveis como a autoeficácia, suporte social e necessidades psicológicas básicas não foram investigadas.

Apesar das limitações apresentadas, este trabalho evidencia as consequências perceptivas e conceituais da atividade física, ao considerar as disposições motivacionais na manutenção do exercício e suas consequências. Corrobora com uma lacuna importante na literatura, considerando que os processos afetivos que determinam a adoção e manutenção do comportamento frente ao exercício tem recebido consideravelmente menos atenção ${ }^{21}$.

$\mathrm{Na}$ perspectiva de saúde, entender as consequências da manutenção da atividade física é crucial. A extensão às percepções positivas de saúde é conduzida na medida que o indivíduo se percebe mais profundamente satisfeito com a prática, implicando assim, na manutenção ${ }^{30}$. Esse entendimento é importante para evitar generalizações na relação atividade física e saúde.

Diante disso, considera-se que a relação entre a manutenção e a percepção positiva de saúde pode serbidi- recional, ao identificar os referidos benefícios os adolescentes adotam comportamentos mais autônomos e autodeterminado com a prática. Para isso, estudos longitudinais são requeridos, afim de examinar os condicionantes intrapessoais que determinam a manutenção da prática de exercícios e consequente a melhora na percepção de saúde. Conclui-se que adolescentes no estágio de manutenção para a prática de exercícios perceberam-se com melhores condições de saúde comparados àqueles que não praticaram exercícios. As moças no estágio de manutenção apresentaram-se mais satisfeitas com o peso corporal, além de indicarem maior autocontrole do estresse. O conjunto dessas percepções positivas, associadas à prática de exercício parece representar uma melhor disposição psicossocial destes adolescentes. A percepção de benefício à saúde esteve associada ao tempo que estes adolescentes estiveram expostos à atividade física regular, pois, não foram observados benefícios para os adolescentes que praticaram exercícios a menos de seis meses.

\section{Conflito de Interesses}

Os autores declararam não haver nenhum conflito de interesse.

\section{Contribuição dos autores}

Todos os autores participaram com contribuições substanciais para a concepção deste estudo. Matias TS e Andrade A contribuíram com a concepção do projeto, o delineamento do problema de pesquisa, a coordenação da coleta de dados e a revisão crítica do artigo. Dominski FH e Maciel GM atuaram na coleta e organização dos dados, bem como, na redação do manuscrito. Lopes MVV e Matias TS contribuíram com o desenvolvimento do método, realização das análises e interpretação dos resultados, e redação do manuscrito.

\section{Referências}

1. Hills AP, Dengel DR, Lubans DR. Supporting public health priorities: Recommendations for physical education and physical activity promotion in schools. Prog Cardiovasc Dis. 2015;57(4):368-74.

2. Honkinen PL, Suominen SB, Välimaa RS, Helenius HY, Rautava PT. Factors associated with perceived health among 12-year-old school children. Relevance of physical exercise and sense of coherence. Scand J Public Health.2005;33(1):35-41.

3. Vancea LA, Barbosa JMV, Menezes AS, Santos CM, Barros MVG de. Associação entre atividade física e percepção de saúde em adolescentes: Revisão sistemática. Rev Bras Ativ Fís Saúde. 2011;16(3):246-54.

4. Bartel KA, Gradisar M, Williamson P. Protective and risk factors for adolescent sleep: A meta-analytic review. Sleep Med Rev. 2015;21:72-85.

5. Salmon P. Effects of physical exercise on anxiety, depression, and sensitivity to stress: a unifying theory. Clin Psychol Rev. 2001;21(1):33-61. 
6. Babic MJ, Morgan PJ, Plotnikoff RC, Lonsdale C, White RL, Lubans DR. Physical activity and physical self-concept in youth: systematic review and meta-analysis. Sports Med Auckl NZ. 2014; 44(11):1589-1601.

7. Instituto Brasileiro de Geografia e Estatística. Pesquisa nacional de saúde do escolar, 2015. Rio de Janeiro: IBGE; 2016. [citado 2017jul14]Disponível em: https://biblioteca. ibge.gov.br/biblioteca-catalogo?view=detalhes\&id $=297870$.

8. Toseeb U, Brage S, Corder K, Dunn VJ, Jones PB, Owens M, St Clair MC, Sluijs EMF, Goodyer IM. Exercise and depressive symptoms in adolescents: a longitudinal cohort study. JAMA Pediatr. 2014;168(12):1093-100.

9. Pareja-Galeano H, Sanchis-Gomar F, Lucia A. Physical activity and depression: Type of exercise matters [carta]. JAMA Pediatr. 2015;169(3):288-9.

10. Hutchison AJ, Breckon JD, Johnston LH. Physical activity behavior change interventions based on the transtheoretical model: a systematic review. Health Educ Behav. 2009;36(5):829-45.

11. Prochaska JO, Velicer WF. The transtheoretical model of health behavior change. Am J Health Promot. 1997;12(1):38-48.

12. Matias TS, da Silveira Viana M, Kretzer FL, Andrade A. Autodeterminação de adolescentes em diferentes estágios de mudança para o exercício físico. Rev Educ Física UEM. 2014;25(2):211-22.

13. Ersöz G, Eklund RC. Behavioral regulations and dispositional flow in exercise among American college students relative to stages of change and gender. J Am Coll Health. 2017;65(2):94-102.

14. Vancampfort D, Moens H, Madou T, De Backer T, Vallons V, Bruyninx P, Vanheuverzwijn S, Mota CT, Soundy A, Probst M. Autonomous motivation is associated with the maintenance stage of behavior change in people with affective disorders. Psychiatry Res. 2016; 240:267-71.

15. Rolim,M.Auto-eficácia, estilo de vida e desempenho cognitivo de adolescentes ativos e sedentários. [Dissertação]. [Florianópolis]: Universidade do Estado de Santa Catarina; 2007.

16. Dumith SC, Gigante DP, Domingues MR. Stages of change for physical activity in adults from Southern Brazil: a population-based survey. Int J Behav Nutr Phys Act. 2007;4(25)1-10.

17. Herman KM, Hopman WM, Sabiston CM. Physical activity, screen time and self-rated health and mental health in Canadian adolescents. Prev Med. 2015; 73:112-16.

18. Nuviala A, Ruiz Juan F, NuvialaNuviala R. Actividad física y autopercepción de la salud en adolescentes. Pensar En Mov Rev Cienc Ejerc Salud. 2011; 8(1):34-41.
19. Piko BF. Self-perceived health among adolescents: the role of gender and psychosocial factors. Eur J Pediatr. 2007;166(7):701-08.

20. Gísladóttir TL, Matthíasdóttir A, Kristjánsdóttir H. The effect of adolescents' sports clubs participation on self-reported mental and physical conditions and future expectations. J Sports Sci. 2013;31(10):1139-45.

21. Williams DM. Exercise, affect, and adherence: an integrated model and a case for self-paced exercise. J Sport Exerc Psychol. 2008; 30(5):471-96.

22. Rosa C, Bicudo LFP, Vaisberg MW. Influências do exercício na resposta imune. Rev Bras Med Esporte. 2002;8(4):167-72.

23. Gleeson M, Bishop NC, Stensel DJ, Lindley MR, Mastana SS, Nimmo MA. The anti-inflammatory effects of exercise: mechanisms and implications for the prevention and treatment of disease. Nat Rev Immunol. 2011; 11(9):607-15.

24. Matias TS, Rolim MKSB, Kretzer FL, Schmoelz CP, Andrade A. Corporal satisfaction associated with physical activity practice during adolescence. Mot J Phys Educ UNESP. 2010;16(2):370-78.

25. Hausenblas HA, Fallon EA. Exercise and body image: A meta-analysis. Psychol Health. 2006;21(1):33-47.

26. Campbell A, Hausenblas HA. Effects of exercise interventions on body image: A meta-analysis. J Health Psychol. 2009;14(6):780-93.

27. Bandura A. The anatomy of stages of change [editorial]. Am J Health Promot. 1997;12(1):8-10.

28. Chatzisarantis NL, Hagger MS. The moral worth of sport reconsidered: Contributions of recreational sport and competitive sport to life aspirations and psychological wellbeing. J Sports Sci. 2007;25(9):1047-56.

29. Birkeland MS, Torsheim T, Wold B. A longitudinal study of the relationship between leisure-time physical activity and depressed mood among adolescents. Psychol Sport Exerc. 2009;10(1):25-34.

30. Ryan RM. Self-determination theory and physical activity: the dynamics of motivation in development and wellness. Hell J Psychol. 2009;6(2):107-24. 